\title{
Whole pelvic intensity-modulated radiotherapy for high- risk prostate cancer: a preliminary report
}

\author{
Ji Hyeon Joo, MD', Yeon Joo Kim, MD1', Young Seok Kim, MD', Eun Kyung Choi, MD', \\ Jong Hoon Kim, MD', Sang-wook Lee, MD', Si Yeol Song, MD', Sang Min Yoon, MD', \\ Su Ssan Kim, MD', Jin-hong Park, MD'1, Yuri Jeong, MD', Hanjong Ahn, MD², \\ Choung-Soo Kim, MD², Jae-Lyun Lee, MD³, Seung Do Ahn, MD'
}

Departments of ${ }^{1}$ Radiation Oncology, ${ }^{2}$ Urology, and ${ }^{3}$ Oncology, Asan Medical Center, University of Ulsan College of Medicine, Seoul, Korea

Purpose: To assess the clinical efficacy and toxicity of whole pelvic intensity-modulated radiotherapy (WP-IMRT) for high-risk prostate cancer.

Materials and Methods: Patients with high-risk prostate cancer treated between 2008 and 2013 were reviewed. The study included patients who had undergone WP-IMRT with image guidance using electronic portal imaging devices and/or cone-beam computed tomography. The endorectal balloon was used in $93 \%$ of patients. Patients received either 46 Gy to the whole pelvis plus a boost of up to 76 Gy to the prostate in 2 Gy daily fractions, or 44 Gy to the whole pelvis plus a boost of up to 72.6 Gy to the prostate in $2.2 \mathrm{~Gy}$ fractions.

Results: The study cohort included 70 patients, of whom $55(78 \%)$ had a Gleason score of 8 to 10 and $50(71 \%)$ had a prostatespecific antigen level $>20 \mathrm{ng} / \mathrm{mL}$. The androgen deprivation therapy was combined in 62 patients. The biochemical failure-free survival rate was $86.7 \%$ at 2 years. Acute any grade gastrointestinal (GI) and genitourinary (GU) toxicity rates were $47 \%$ and $73 \%$, respectively. The actuarial rate of late grade 2 or worse toxicity at 2 years was $12.9 \%$ for $\mathrm{Gl}$, and $5.7 \%$ for $\mathrm{GU}$ with no late grade 4 toxicity.

Conclusion: WP-IMRT was well tolerated with no severe acute or late toxicities, resulting in at least similar biochemical control to that of the historic control group with a small field. The long-term efficacy and toxicity will be assessed in the future, and a prospective randomized trial is needed to verify these findings.

Keywords: Prostate neoplasms, Intensity-modulated radiotherapy, Complications

\section{Introduction}

The appropriate extent of the radiation field remains controversial, especially in patients with high-risk prostate cancer. The probability of lymph node metastasis is considerable even in patients with negative imaging studies [1,2]. Therefore, the effect of extended field radiotherapy (RT), which includes the whole pelvis, was tested in large prospective trials in high-risk patients. Comparisons of the efficacy of whole pelvic RT (WPRT) to that of prostate-only RT (PORT) showed that the long-term

Received 16 September 2013, Revised 5 October 2013, Accepted 18 October 2013.

Correspondence: Young Seok Kim, MD, Department of Radiation Oncology, Asan Medical Center, University of Ulsan College of Medicine, 88 Olympic-ro 43-gil, Songpa-gu, Seoul 138-736, Korea. Tel: +82-2-3010-5614, Fax: +82-2-3010-6950, E-mail: ysk@amc.seoul.kr

(c) This is an Open Access article distributed under the terms of the Creative Commons Attribution Non-Commercial License (http://creativecommons.org/ licenses/by-nc/3.0/) which permits unrestricted non-commercial use, distribution, and reproduction in any medium, provided the original work is properly cited.

www.e-roj.org 
progression-free survival rates were similar in both arms [3-5]. However, these studies were designed before the publication of dose-escalation studies [6-9], and the pelvic radiation field was insufficient. In the dose-escalation era, the superiority of WPRT over PORT was confirmed in a large retrospective study in which the application of 75.6 Gy to the prostate resulted in a better biochemical disease control rate in the WPRT group than in patients receiving PORT [10].

The use of WPRT consequently raised concerns regarding the increased incidence of radiation-related toxicities. Acute gastrointestinal (GI) and genitourinary (GU) complications were more frequently reported in patients treated with WPRT than in those receiving PORT $[11,12]$. Regarding late complications, small but insignificant increases were noted in most series $[6,9,10,13]$. Dosimetric studies revealed that intensity-modulated radiotherapy (IMRT) significantly reduced the volume of the bladder and rectum irradiated with high doses in men receiving WPRT [14-18]. Accordingly, acute GI [17] and acute GU [18] toxicities were reduced with the use of IMRT.

In our institution, high-risk prostate cancer patients were treated with dose escalated WPRT. IMRT, endorectal balloon (ERB), and image guidance were used to reduce acute and late toxicities. The primary endpoint of the present study was to assess the clinical efficacy of WPRT in terms of biochemical failure-free survival. The secondary endpoint was to evaluate radiation-related toxicities.

\section{Materials and Methods}

The treatment records of biopsy proven prostate adenocarcinoma patients treated with definitive aim between 2008 and 2013 were reviewed. A total of 83 consecutive, high-risk patients received whole pelvic IMRT (WP-IMRT). Of these, 13 were excluded for the following reasons: follow-up period $<6$ months $(n=10)$, Roach score $<15 \%(n=2)$, or lost to followup $(n=1)$. Of the remaining 70 patients, those meeting any of the following criteria were included in the analysis: clinical T3a stage, or Gleason score (GS) 8-10, or initial prostate-specific antigen (PSA) level $>20 \mathrm{ng} / \mathrm{mL}$. These are high-risk factors for recurrence according to the National Comprehensive Cancer Network guidelines [19]. Although high risk, two patients with Roach score $<15 \%$ were excluded. The pretreatment evaluation consisted of a complete medical history, magnetic resonance imaging (MRI), bone scan, and laboratory tests including PSA.

Computed tomography (CT) simulation was performed with the patients in the supine position with ankle immobilization. The region from the second lumbar vertebrae to the proximal one-third of the femur was scanned with a slice thickness of 2.5 $\mathrm{mm}$. Patients were instructed to empty their bowel and bladder immediately before simulation and subsequent treatment sessions. To reduce the volume of rectum irradiated, and to fix the prostate effectively, the ERB was used for all patients starting in 2009. The details of the balloon and procedure were described previously by D'Amico et al. [20]. Briefly, a homemade rectal balloon was inserted and inflated with $60 \mathrm{~mL}$ of air. The inflated balloon had a diameter of approximately 40 $\mathrm{mm}$ and a length of approximately $60 \mathrm{~mm}$. The gross target volume (GTV) included the whole prostate and the involved lymph nodes. The clinical target volume (CTV) included the GTV, seminal vesicles, and internal iliac, external iliac, and obturator nodal regions. The upper limit of the CTV was the level of the common iliac bifurcation, which was generally located at or just above the L5/S1 junction. The planning target volume was a 5 to $7 \mathrm{~mm}$ expansion of the CTV. After pelvic $R T$, the boost treatment included only the GTV, seminal vesicles, and metastatic lymph nodes. Cone-beam CT (CBCT) for image guidance was performed daily after 2011 to improve the setup stability and ERB localization. CBCT imaging results were examined daily by a physician, focusing on GTV volume and the anterior rectal wall. All patients were treated using WP-IMRT. Doses and fractionation schemes were modified during the study period as follows: before 2011, the whole pelvis and the boost doses were $46 \mathrm{~Gy}$ and $76 \mathrm{~Gy}$, respectively, administered in 2 Gy fractions. After 2011, the fraction size was increased to $2.2 \mathrm{~Gy}$, and the whole pelvis and boost doses were $44 \mathrm{~Gy}$ and $72.6 \mathrm{~Gy}$, respectively. IMRT schemes using five to seven fields were created using Eclipse 10.0 (Varian Medical Systems, Palo Alto, CA, USA). Androgen deprivation therapy (ADT) was administered to the majority of the patients at the discretion of the referring urologists.

PSA level was assessed within 3 months after RT, every 3 months for the next 2 years, and every 6 months thereafter. Biochemical failure was defined as an increase in the PSA level of $2 \mathrm{ng} / \mathrm{mL}$ or more above the PSA nadir after RT, according to the Phoenix definition [21]. Local recurrence was defined as disease recurrence detected in imaging studies, including CT or MRI. The patients were examined weekly during the course of treatment. Acute complications were those occurring within 3 months after treatment and late complications were those occurring after 3 months of treatment. Toxicities were recorded using the National Cancer Institute's Common Terminology 
Criteria for Adverse Events (CTCAE) ver. 4.02, with the addition of the toxicity criteria of the Radiation Therapy Oncology Group (RTOG) morbidity grading scale.

Survival and the incidence of late toxicity were analyzed using the Kaplan-Meier method. Acute toxicity was expressed as a crude rate. The univariate prognostic factor analysis performed using the log-rank test included the following variables: patient age, T \& N stage, GS, pretreatment PSA, PSA nadir, and the use of hormonal therapy. All statistical analyses were performed using SPSS ver. 21 software (IBM, Armonk, NY, USA).

\section{Results}

The characteristics of the 70 patients analyzed are listed in Table 1. Of the 70 patients in the study cohort, 51 received up to $72.6 \mathrm{~Gy}$ in daily fractions of $2.2 \mathrm{~Gy}$ to the prostate and 13 received up to $76 \mathrm{~Gy}$ in daily fractions of $2 \mathrm{~Gy}$ to the prostate. In the remaining six patients, the whole pelvic doses were 46 $\mathrm{Gy}$, but the prostate doses were heterogeneous, ranging from 70 to 80.5 Gy. ADT was administered in 62 patients in the following sequences: neoadjuvant + concurrent + adjuvant $(n=39)$, concurrent + adjuvant $(n=16)$, neoadjuvant $(n$

Table 1. Patient characteristics

\begin{tabular}{lc}
\hline \multicolumn{1}{c}{ Characteristic } & No. of patients (\%) \\
\hline Age (yr), mean (range) & $72(52-81)$ \\
Comorbidity & $48(69)$ \\
Yes & $22(31)$ \\
No & \\
T stage & $25(36)$ \\
T1-2c & $45(64)$ \\
T3a-4 & \\
N stage & $56(80)$ \\
No & $14(20)$ \\
N1 & \\
Gleason score & $2(3)$ \\
$\leq 6$ & $12(17)$ \\
7 & $55(78)$ \\
$8-10$ & \\
Initial PSA level (ng/mL) & $11(16)$ \\
$\leq 10$ & $9(13)$ \\
$10-20$ & $50(71)$ \\
$>20$ & \\
ADT & $62(89)$ \\
Yes & $8(11)$ \\
No &
\end{tabular}

PSA, prostate specific antigen; ADT, androgen deprivation therapy.
$=5)$, concurrent $(n=1)$, and adjuvant $(n=1)$. The median duration of hormonal therapy was 16 months (range, 5 to 60 months). Total androgen blockade was used in 49 patients and gonadotropin-releasing hormone agonist alone in 13.

The median follow-up duration was 19 months (range, 2 to 61 months). At 2 years, the biochemical failure-free survival, local recurrence-free survival, and distant metastasis-free survival rates were $86.7 \%, 93.0 \%$, and $92.9 \%$, respectively. The disease-free survival and overall survival rates were $87.6 \%$ and $91.8 \%$, respectively. In the prognostic analysis for biochemical failure-free survival, the T/N stage and PSA nadir had $p$-values $\leq 0.05$. The results of the prognostic factor analysis are summarized in Table 2.

The numbers of patients with acute and late toxicities and their grades are listed in Table 3. RT was well tolerated with no severe toxicity. Acute GI and GU toxicities of any grades were observed in $47 \%$ and $73 \%$ of patients. None of the patients experienced grade 3 acute GI toxicity. The symptoms of $\mathrm{Gl}$ toxicity were anorexia $(n=10)$, abdominal pain $(n=9)$, dyspepsia $(n=8)$, diarrhea $(n=7)$, rectal discomfort $(n=5)$, abdominal distension $(n=3)$, and nausea $(n=1)$. The only grade 2 symptom was diarrhea requiring medication. The only

Table 2. Univariate analysis of patient characteristics for BFS

\begin{tabular}{|c|c|c|c|}
\hline Variable & No. of patients & $2-y r$ BFS (\%) & $p$-value \\
\hline Age (yr) & & & 0.95 \\
\hline$<75$ & 46 & 85 & \\
\hline$\geq 75$ & 24 & 89 & \\
\hline T stage & & & 0.03 \\
\hline T1-2c & 25 & 100 & \\
\hline Т3a-4 & 45 & 79.1 & \\
\hline N stage & & & $<0.01$ \\
\hline No & 56 & 95.5 & \\
\hline N1 & 14 & 52.4 & \\
\hline Gleason score & & & 0.38 \\
\hline $6-7$ & 14 & 92.9 & \\
\hline $8-10$ & 55 & 83.7 & \\
\hline ¡PSA & & & 0.87 \\
\hline$\leq 20$ & 20 & 89.7 & \\
\hline$>20$ & 50 & 85.4 & \\
\hline PSA nadir & & & $<0.01$ \\
\hline$<0.2$ & 59 & 94.9 & \\
\hline$\geq 0.2$ & 11 & 48.5 & \\
\hline ADT & & & 0.26 \\
\hline No & 8 & 100 & \\
\hline Yes & 62 & 84.3 & \\
\hline
\end{tabular}

BFS, biochemical failure-free survival; iPSA, initial prostate specific antigen; ADT, androgen deprivation therapy. 
acute grade $3 \mathrm{GU}$ toxicity was an increase in urinary frequency, with voiding intervals of less than 1 hour. Medication was needed until the completion of RT. Other grade 1-2 acute urinary symptoms included nocturia $(n=30)$, increased urinary frequency $(n=21)$, dysuria $(n=20)$, urgency $(n=$ 13), incontinence $(n=3)$, and hematuria $(n=2)$. Overall, the incidence rates of late $\mathrm{GI}$ and $\mathrm{GU}$ toxicity of any grade were 16\% and 30\%, respectively. Regarding late Gl toxicity, grade 3 radiation proctitis was diagnosed in three patients who showed repeated blood tinged stool requiring transfusion $(n=2)$ or argon plasma coagulation $(n=1)$. Regarding late GU toxicity, the only grade 3 toxicity was gross hematuria. Cystoscopic evaluation showed radiation induced mucosal changes. The rate of late grade 2 or worse toxicity at 2 years was $12.9 \%$ for $\mathrm{GI}$ and $5.7 \%$ for GU. None of the patients experienced late grade 4 toxicity.

\section{Discussion and Conclusion}

The present study was a preliminary analysis of the effects of WP-IMRT in high-risk prostate cancer patients. The results showed acceptable outcomes in terms of biochemical control and toxicity profiles, although further studies with long-term follow-up are necessary to verify these results. The results of previous studies using PORT in high-risk patients are summarized in Table 4. A study from the MD Anderson Cancer Center showed a 5-year biochemical failure-free survival of $82 \%$ in patients treated with 75.6 Gy plus ADT for $\geq 2$ years

Table 3. Acute and late toxicities

\begin{tabular}{lcccc}
\hline Toxicity & Grade 1 & Grade 2 & Grade 3 & Total \\
\hline Acute GI & $32(45)$ & $1(2)$ & 0 & $33(47)$ \\
Acute GU & $35(50)$ & $15(21)$ & $1(2)$ & $51(73)$ \\
Late GI & $6(9)$ & $2(3)$ & $3(4)$ & $11(16)$ \\
Late GU & $17(24)$ & $3(4)$ & $1(2)$ & $21(30)$ \\
\hline
\end{tabular}

Values are presented as number (\%).

$\mathrm{Gl}$, gastrointestinal; GU, genitourinary.
[22]. Other trials that included patients treated with higher radiation doses (>75 Gy) $[10,23]$ showed 5 -year biochemical control rates of approximately 70\%. In the present study, the biochemical control rate in high-risk patients was $86.7 \%$ at 2 years. Considering that the present study included a higher proportion of patients with stage T3 prostate cancer, GS 8-10 and PSA $>20 \mathrm{ng} / \mathrm{mL}$ than previous studies [10,22,23], our results can be interpreted as at least comparable or better than those of previously published studies. However, the present work is a preliminary report with short-term followup, and drawing definitive conclusions is therefore difficult. Nevertheless, the patient outcomes in the present study were comparable to those of previous studies possibly because of the use of an adequate radiation field and the frequent use of combined hormonal therapy. The relation between elective pelvic node irradiation and PSA relapse is controversial. In the RTOG 9413 trial, WPRT showed a statistically significant benefit over PORT, as demonstrated in the 4-year progressionfree survival (54\% vs. 47\%, $p=0.02$ ); however, a significant difference was no longer observed in the long-term update $[3,4]$. In another prospective study, GETUG-01, prostate cancer patients were randomized to WPRT or PORT treatment groups, and no differences in 5-year progression-free survival were observed (66\% in WPRT vs. 65\% in PORT, p = 0.34). Because the definition of progression in the RTOG 9413 trial included death from any cause, non-prostate cancer-related deaths may have dominated over prostate cancer-related events in the long-term follow-up. In the GEUTG-01 study, more than $50 \%$ of the patients had a risk of nodal involvement of $<15 \%$ and the upper limit of the radiation field was relatively low $(\mathrm{S} 1-2)$. Furthermore, these trials were designed before the publication of dose-escalation studies: in RTOG 9413, the prescribed doses to the pelvis and prostate were $50.4 \mathrm{~Gy}$ and 70.2 Gy, respectively, and in GETUG-01, they were 46 Gy and 66 to $70 \mathrm{~Gy}$, respectively. High-dose RT, which is currently in use, was tested in a comparative setting in a retrospective study conducted at Yale University in which the effects of WPRT and PORT were compared in patients with a Roach score

Table 4. Prostate-only radiotherapy and BFS in high-risk patients

\begin{tabular}{|c|c|c|c|c|}
\hline Author & Patient & Endpoint & Radiation dose (Gy) & BFS $(\%)$ \\
\hline Nguyen et al. [22] & High-risk & $5-y r$ BFS & $\geq 75.6$ & $82.0($ ADT+)/73.9 (ADT-) \\
\hline Kuban et al. [23] & High-risk & $5 / 10-y r$ BFS & 78 & $72 / 67$ \\
\hline Aizer et al. [10] & Roach >15\% & $5-y r$ BFS & 75.6 & 69 \\
\hline Zelefsky et al. [38] & High-risk & 5/7-yr BFS & $70.2-86.4$ & $62 / 54$ \\
\hline
\end{tabular}

BFS, biochemical failure-free survival; ADT, androgen deprivation therapy. 
$>15 \%$. The prescribed dose in this study was $75.6 \mathrm{~Gy}$ in $1.8 \mathrm{~Gy}$ fractions. When the $\alpha / \beta$ ratio of a prostate tumor is assumed to be 2 , the calculated equivalent dose in $2 \mathrm{~Gy}$ fractions $\left(\mathrm{EQD}_{2}\right)$ is $71.8 \mathrm{~Gy}$. In the Yale study, the four-field box technique was used for the treatment of the whole pelvis and the 4-year biochemical failure-free survival rates for patients receiving WPRT and PORT were 86\% and 69\%, respectively $(p<0.01)$ [10]. In the present study, the irradiated dose was changed from 76 Gy in 2 Gy fractions to 72.6 Gy in 2.2 Gy fractions $\left(\mathrm{EQD}_{2}=76.2\right.$ Gy), and IMRT was used in all treatment plans. In addition, the follow-up period was shorter than that of the Yale study. Otherwise, the patient population and treatment regimens were similar to those of the whole pelvis arm in the Yale study, and both studies had similar outcomes. Although we did not include a control group and longer follow-up is needed, the biochemical control in the present study was comparable to that reported in previous studies. However, the differences in the follow-up duration make a direct comparison difficult. Our results suggest that patients with high-risk prostate cancer in whom the predicted risk of nodal involvement exceeds 15\% may benefit from elective pelvic nodal irradiation in terms of biochemical control. However, additional studies with longer follow-up are necessary to draw definitive conclusions.

During the treatment period, only one patient experienced grade $2 \mathrm{Gl}$ toxicity (diarrhea), and no grade 3 toxicities were reported. Acute grade 2 and grade $3 \mathrm{GU}$ toxicities were reported in 15 patients and one patient, respectively. Anorexia and nocturia were the most frequent symptoms and they resolved spontaneously during follow-up. An association between IMRT and a reduction in the incidence of acute complications has been reported previously. In a study conducted at Memorial Sloan-Kettering Cancer Center, Ashman et al. [17] compared dosimetric outcomes and toxicities in 13 patients treated with two-dimensional, three-dimensional conventional RT (3D-CRT), and IMRT and showed that the bowel volume receiving more than 45 Gy (V45), the mean bowel dose, rectal V45, and bladder V45 were all significantly reduced with IMRT. The acute $\mathrm{GI}$ and $\mathrm{GU}$ toxicities were minimal with no case of grade 3 or higher toxicity. In the present study, the rate of late grade 2 or worse toxicity at 2 years was $12.9 \%$ for GI and $5.7 \%$ for GU toxicities, which is a better result than that of the RTOG 9413 whole-pelvic group, which used lower radiation doses administered using the 4-field technique. In that study, the rate of 5-year $\mathrm{Gl}$ and $\mathrm{GU}$ toxicities $\geq$ grade 2 were $15.2 \%$ and 14.9\%, respectively [24]. Quon et al. [25] analyzed the toxicity rates of dose escalated WPRT using a whole-pelvic dose of
$45 \mathrm{~Gy}$ in $1.8 \mathrm{~Gy}$ fractions and a concomitant 22.5 Gy prostate IMRT boost. The 4-year GI and GU toxicities $\geq$ grade 2 were $5.8 \%$ and $10.1 \%$, respectively. Compared to the data from our institution, the $E Q D_{2}$ was lower for the pelvis and higher for the prostate boost [25], which may have contributed to the higher $\mathrm{Gl}$ and lower GU late toxicities in the present study. An association between the prostate dose and chronic GU toxicity has been suggested in previous studies $[26,27]$. The reported late $\mathrm{Gl}$ and $\mathrm{GU}$ toxicity rates in dose escalated PORT studies are $5 \%$ to $46 \%$ and $11 \%$ to $39 \%$, respectively [28-31]. Generally, most late rectal toxicities attributed to RT appear 12 to 18 months after completion of treatment. However, this issue is controversial. In the Medical Research Council RT01 trial, patients were treated with 64 to $74 \mathrm{~Gy}$ and the 2- and 5-year cumulative proportion of patients showing treatment-related toxicities was reported. Grade 2 or higher GI toxicity rates at 2 and 5 years were $14 \%$ and 24\%, respectively. Although bowel function complications developed mostly within 2 years, with a prevalence of 6 to 24 months, other GI and GU complications increased after 2 years. The GU toxicity rates at 2 and 5 years were $6 \%$ and $8 \%$, respectively [31]. The later onset of late $\mathrm{GU}$ toxicity compared to $\mathrm{GI}$ toxicity was analyzed in several studies. Zelefsky et al. [26] used a dose of 66 to 81 Gy and a median follow-up of 10 years and showed that the median time to development of GU symptoms of grade $\geq 2$ was 30 months, compared with 17 months for patients with $\mathrm{Gl}$ side effects of grade $\geq 2$. Gardner et al. [32] used 77.4 Gy and showed that the actuarial risk of grade $\geq 2$ hematuria was $21 \%$ at 5 years and $47 \%$ at 15 years; the incidence of GU toxicities increased progressively during the entire study period, which had a median follow-up duration of 13.1 years. Therefore, longer follow-up may not worsen our $\mathrm{Gl}$ complication results, whereas further follow-up for late GU complications will be needed considering the different time trends of late $\mathrm{GI}$ and $\mathrm{GU}$ toxicities. IMRT was shown to lower the rate of late toxicities in high-dose prostate RT. Zelefsky et al. [26] compared the long-term tolerance of 3D-CRT and IMRT and showed that IMRT reduced the rate of GI toxicity $\geq$ grade 2 by $8 \%$ (13\% to $5 \%, p<0.01)$ at the 10 -year follow-up. The rate of 10 -year GU toxicity $\geq$ grade 2 in patients treated with IMRT was 17\% [33]. The prostate gland immobilization and rectal sparing effects of the ERB were tested by several investigators [34]. When combined with IMRT, it reduced prostate motion and had a favorable rectal toxicity profile [35,36]. Zelefsky et al. [37] showed the benefits of the direct visualization of the pelvic anatomy. In the present study, we used CBCT in all treatments 
for proper setup and rectal sparing.

The present study had several limitations. Despite the large number of patients included, the study was limited by its retrospective design, the heterogeneous use of ADT and a short follow-up period. Therefore, the authors will assess the long-term efficacy and toxicity again in the future to verify the benefits of WP-IMRT for the treatment of high-risk prostate cancer patients.

In conclusion, WP-IMRT was well tolerated with no severe acute or late toxicities. The present preliminary report shows that WP-IMRT results in acceptable biochemical control rates compared to those reported previously with a small field. The long-term efficacy and toxicity will be assessed again in the future, and a prospective randomized trial is needed to verify the present findings.

\section{Conflict of Interest}

No potential conflict of interest relevant to this article was reported.

\section{References}

1. Abuzallouf S, Dayes I, Lukka H. Baseline staging of newly diagnosed prostate cancer: a summary of the literature. J Urol 2004;171(6 Pt 1):2122-7.

2. Tiguert R, Gheiler EL, Tefilli MV, et al. Lymph node size does not correlate with the presence of prostate cancer metastasis. Urology 1999:53:367-71.

3. Roach M 3rd, DeSilvio M, Lawton $C$, et al. Phase III trial comparing whole-pelvic versus prostate-only radiotherapy and neoadjuvant versus adjuvant combined androgen suppression: Radiation Therapy Oncology Group 9413. J Clin Oncol 2003;21:1904-11.

4. Lawton CA, DeSilvio M, Roach M 3rd, et al. An update of the phase III trial comparing whole pelvic to prostate only radiotherapy and neoadjuvant to adjuvant total androgen suppression: updated analysis of RTOG 94-13, with emphasis on unexpected hormone/radiation interactions. Int J Radiat Oncol Biol Phys 2007;69:646-55.

5. Pommier $P$, Chabaud $S$, Lagrange $J$, et al. Is there a role for pelvic irradiation in localized prostate adenocarcinoma? Preliminary results of GETUG-01. J Clin Oncol 2007;25:536673.

6. Hanks GE, Hanlon AL, Schultheiss TE, et al. Dose escalation with 3D conformal treatment: five year outcomes, treatment optimization, and future directions. Int J Radiat Oncol Biol Phys 1998;41:501-10.
7. Pollack A, Zagars GK, Smith LG, et al. Preliminary results of a randomized radiotherapy dose-escalation study comparing 70 Gy with 78 Gy for prostate cancer. J Clin Oncol 2000;18:390411.

8. Valicenti R, Lu J, Pilepich M, Asbell S, Grignon D. Survival advantage from higher-dose radiation therapy for clinically localized prostate cancer treated on the Radiation Therapy Oncology Group trials. J Clin Oncol 2000;18:2740-6.

9. Zelefsky MJ, Leibel SA, Gaudin PB, et al. Dose escalation with three-dimensional conformal radiation therapy affects the outcome in prostate cancer. Int J Radiat Oncol Biol Phys 1998; 41:491-500.

10. Aizer AA, Yu JB, McKeon AM, Decker RH, Colberg JW, Peschel RE. Whole pelvic radiotherapy versus prostate only radiotherapy in the management of locally advanced or aggressive prostate adenocarcinoma. Int J Radiat Oncol Biol Phys 2009;75:1344-9.

11. Perez CA, Lee HK, Georgiou A, Lockett MA. Technical factors affecting morbidity in definitive irradiation for localized carcinoma of the prostate. Int J Radiat Oncol Biol Phys 1994; 28:811-9.

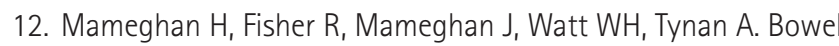
complications after radiotherapy for carcinoma of the prostate: the volume effect. Int J Radiat Oncol Biol Phys 1990;18:31520.

13. Ploysongsang SS, Aron BS, Shehata WM. Radiation therapy in prostate cancer: whole pelvis with prostate boost or small field to prostate? Urology 1992;40:18-26.

14. Nutting CM, Convery DJ, Cosgrove VP, et al. Reduction of smal and large bowel irradiation using an optimized intensitymodulated pelvic radiotherapy technique in patients with prostate cancer. Int J Radiat Oncol Biol Phys 2000;48:649-56.

15. Luxton G, Hancock SL, Boyer AL. Dosimetry and radiobiologic model comparison of IMRT and 3D conformal radiotherapy in treatment of carcinoma of the prostate. Int J Radiat Oncol Biol Phys 2004;59:267-84.

16. Cavey ML, Bayouth JE, Colman M, Endres EJ, Sanguineti G. IMRT to escalate the dose to the prostate while treating the pelvic nodes. Strahlenther Onkol 2005;181:431-41.

17. Ashman JB, Zelefsky MJ, Hunt MS, Leibel SA, Fuks Z. Whole pelvic radiotherapy for prostate cancer using $3 \mathrm{D}$ conformal and intensity-modulated radiotherapy. Int J Radiat Oncol Biol Phys 2005;63:765-71

18. Jani $A B$, Su A, Milano MT. Intensity-modulated versus conventional pelvic radiotherapy for prostate cancer: analysis of acute toxicity. Urology 2006;67:147-51.

19. National Comprehensive Cancer Network. NCCN Guidelines(R) Updates. J Natl Compr Canc Netw 2013;11:xxxii-xxxvi.

20. D'Amico AV, Manola J, Loffredo M, et al. A practical method to achieve prostate gland immobilization and target verification for daily treatment. Int J Radiat Oncol Biol Phys 2001;51:1431- 
6.

21. Roach M 3rd, Hanks G, Thames H Jr, et al. Defining biochemical failure following radiotherapy with or without hormonal therapy in men with clinically localized prostate cancer: recommendations of the RTOG-ASTRO Phoenix Consensus Conference. Int J Radiat Oncol Biol Phys 2006;65:965-74.

22. Nguyen $Q N$, Levy LB, Lee $A K$, et al. Long-term outcomes for men with high-risk prostate cancer treated definitively with external beam radiotherapy with or without androgen deprivation. Cancer 2013;119:3265-71.

23. Kuban $D A$, Levy $L B$, Cheung $M R$, et al. Long-term failure patterns and survival in a randomized dose-escalation trial for prostate cancer. Who dies of disease? Int J Radiat Oncol Biol Phys 2011; 79:1310-7.

24. Roach M 3rd, DeSilvio M, Valicenti $R$, et al. Whole-pelvis, "minipelvis," or prostate-only external beam radiotherapy after neoadjuvant and concurrent hormonal therapy in patients treated in the Radiation Therapy Oncology Group 9413 trial. Int J Radiat Oncol Biol Phys 2006;66:647-53.

25. Quon H, Cheung PC, Loblaw DA, et al. Hypofractionated concomitant intensity-modulated radiotherapy boost for highrisk prostate cancer: late toxicity. Int J Radiat Oncol Biol Phys 2012;82:898-905.

26. Zelefsky MJ, Levin EJ, Hunt M, et al. Incidence of late rectal and urinary toxicities after three-dimensional conformal radiotherapy and intensity-modulated radiotherapy for localized prostate cancer. Int J Radiat Oncol Biol Phys 2008;70:1124-9.

27. Viswanathan AN, Yorke ED, Marks LB, Eifel PJ, Shipley WU. Radiation dose-volume effects of the urinary bladder. Int J Radiat Oncol Biol Phys 2010;76(3 Suppl):S116-22.

28. Pollack A, Zagars GK, Starkschall G, et al. Prostate cancer radiation dose response: results of the $M$. D. Anderson phase III randomized trial. Int J Radiat Oncol Biol Phys 2002;53:1097105.

29. Zietman $A L$, DeSilvio $M L$, Slater JD, et al. Comparison of conventional-dose vs high-dose conformal radiation therapy in clinically localized adenocarcinoma of the prostate: a randomized controlled trial. JAMA 2005;294:1233-9.
30. Peeters ST, Heemsbergen WD, Koper PC, et al. Dose-response in radiotherapy for localized prostate cancer: results of the Dutch multicenter randomized phase III trial comparing $68 \mathrm{~Gy}$ of radiotherapy with 78 Gy. J Clin Oncol 2006;24:1990-6.

31. Dearnaley DP, Sydes MR, Graham JD, et al. Escalated-dose versus standard-dose conformal radiotherapy in prostate cancer: first results from the MRC RT01 randomised controlled trial. Lancet Oncol 2007;8:475-87.

32. Gardner BG, Zietman AL, Shipley WU, Skowronski UE, McManus P. Late normal tissue sequelae in the second decade after high dose radiation therapy with combined photons and conformal protons for locally advanced prostate cancer. J Urol 2002;167: 123-6.

33. Alicikus ZA, Yamada $Y$, Zhang $Z$, et al. Ten-year outcomes of high-dose, intensity-modulated radiotherapy for localized prostate cancer. Cancer 2011;117:1429-37.

34. van Lin EN, van der Vight LP, Witjes JA, Huisman HJ, Leer JW, Visser AG. The effect of an endorectal balloon and off-line correction on the interfraction systematic and random prostate position variations: a comparative study. Int J Radiat Oncol Biol Phys 2005;61:278-88.

35. Bastasch MD, Teh BS, Mai WY, McGary JE, Grant WH 3rd, Butler EB. Tolerance of endorectal balloon in 396 patients treated with intensity-modulated radiation therapy (IMRT) for prostate cancer. Am J Clin Oncol 2006;29:8-11.

36. Teh BS, McGary JE, Dong $L$, et al. The use of rectal balloon during the delivery of intensity modulated radiotherapy (IMRT) for prostate cancer: more than just a prostate gland immobilization device? Cancer J 2002;8:476-83.

37. Zelefsky MJ, Kollmeier M, Cox B, et al. Improved clinical outcomes with high-dose image guided radiotherapy compared with non-IGRT for the treatment of clinically localized prostate cancer. Int J Radiat Oncol Biol Phys 2012;84:125-9.

38. Zelefsky MJ, Yamada Y, Fuks Z, et al. Long-term results of conformal radiotherapy for prostate cancer: impact of doseescalation on biochemical tumor control and distant metastases-free survival outcomes. Int J Radiat Oncol Biol Phys 2008;71:1028-33. 